\title{
Lesson of the month 2: The limitations of steroid therapy in bradykinin-mediated angioedema attacks
}

\author{
Authors: Sharif Ismail, ${ }^{A}$ Leo Cheng, ${ }^{B}$ Sofia Grigoriadou, ${ }^{C}$ James Laffan ${ }^{D}$ and Manoj Menon ${ }^{E}$
}

\begin{abstract}
Acute angioedema attacks are conventionally treated with antihistamines and steroids, in line with a presumed mechanism of disease involving overwhelming mast-cell degranulation. This approach overlooks a small but important minority of cases in which attacks are bradykinin driven and exhibit poor responsiveness to steroid or anti-histamine therapy. These patients may have a family history of angioedema (hereditary angioedema), or a past medical history including B-cell lymphoproliferative disorders or autoimmune disease (acquired angioedema). Rather than steroid therapy, they respond to administration of a bradykinin inhibitor, or more commonly, a C1 esterase inhibitor substitute, to control acute symptoms and reduce the probability of invasive airway insertion. In the longterm, they require $\mathrm{C} 1$ esterase inhibitor sparing therapy and a treat-the-cause approach to reduce the risk of recurrent attacks. We present here a case of a middle-aged woman who presented with recurrent angioedema of initially uncertain aetiology.
\end{abstract}

KEYWORDS: Angioedema, bradykinin, steroid, C1 esterase inhibitor

\section{Case history}

A 57-year-old woman of Afro-Caribbean origin presented to A\&E with facial swelling possibly triggered by an insect bite which resolved within 48 hours with Piriton (chlorphenamine), IM adrenaline and IV hydrocortisone therapy. Four weeks later she presented again with progressive onset difficulty in breathing and mouth swelling. Her past medical history included hypertension controlled with bendroflumethiazide (no previous angiotensin-converting-enzyme (ACE) inhibitor use) and low-grade non-Hodgkin lymphoma, diagnosed around six months previously and not under active treatment.

On this second occasion, facial and oral mucosal swelling and secretions worsened over 6-8 hours despite Piriton, IM

Authors: ${ }^{A}$ academic foundation year 2 doctor, Royal London Hospital, London, UK; ${ }^{\mathrm{B}}$ consultant in oral, maxillofacial and head and neck surgery, St Bartholomew's, Royal London and Homerton University Hospitals, London, UK; ' Consultant in immunology, Royal

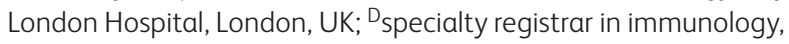
Royal London Hospital, London, UK; ${ }^{E}$ consultant in respiratory medicine, Homerton University Hospital, London, UK adrenaline, IV steroids and nebulised-adrenaline therapy. The patient required intubation due to airway compromise and spent four weeks in intensive care, during which she was treated with high-dose dexamethasone and chlorphenamine, but subsequent re-evaluations of her oropharynx, nasopharynx and larynx with flexible nasendoscope demonstrated persistent soft-tissue swelling that was slow to resolve. Surgical tracheostomy was initially delayed due to profuse secretions, an enlarged thyroid nodule and extensive upper airway oedema with possible tracheal mucosal oedema, but was eventually performed and regular direct inspections of the airway with flexible nasendoscope were carried out. The tracheostomy tube was downsized from an adjustable flange tube to smaller fixed length tracheostomy tube following repeated nasendoscopic assessments of the airway and upper gastrointestional tract, and the patient was decannulated a month after the tracheostomy was inserted.

Investigations included autoantibody (tissue transglutaminase, smooth muscle, anti-mitochondrial, antinuclear antibody (ANA) and anti-neutrophil cytoplasmic antibodies (ANCA)) and virology screens (HIV, hepatitis B and hepatitis C) which were negative. Tryptase was normal on both occasions measured ( 5 hours and 24 hours after symptoms onset) thereby making incipient anaphylaxis or mastocytosis as the cause of the presentation unlikely. Complement testing results (Table 1) were consistent with $\mathrm{C} 1$ esterase inhibitor (C1-INH) deficiency. Bloods during admission showed progressive lymphocytosis in keeping with diagnosed B-cell lymphoproliferative disorder (Table 2).

The clinical history and investigation results suggested an acquired angioedema, and the patient was commenced on tranexamic acid and given two icatibant prefilled syringes for management of acute attacks. She was advised to present to A\&E immediately if she noticed signs of a recurrence of her symptoms, and was referred to specialist immunology care. She re-presented on two further occasions with difficulty in breathing, hoarseness and retained secretions, requiring further icatibant administration. However, on each occasion, symptoms resolved spontaneously, without airway compromise.

\section{Discussion}

This case demonstrates the importance of considering rare differential diagnoses for acute severe angioedema early in the clinical course. Angioedema is characterised by localised, 
Table 1. Results of complement-testing panel for the patient.

$\begin{array}{llll}\text { Parameter } & \text { Point of testing } & \text { Value } & \text { Hospital normal range } \\ \text { C1EI/C1 inhibitor } & 17 \text { days after admission } & 54 \mathrm{mg} / \mathrm{l} & 220-390 \mathrm{mg} / \mathrm{l} \\ \text { C1E1/C1 inhibitor } & 28 \text { days after admission } & 208 \mathrm{mg} / \mathrm{l} & 220-390 \mathrm{mg} / \mathrm{l} \\ \text { C1q } & 17 \text { days after admission } & 49 \mathrm{mg} / \mathrm{ml} & 118-244 \mathrm{mg} / \mathrm{l} \\ \text { C4 } & 17 \text { days after admission } & <0.03 \mathrm{~g} / \mathrm{l} & 0.16-0.47 \mathrm{~g} / \mathrm{l} \\ \text { C3 } & 7 \text { days after admission } & 0.8 \mathrm{~g} / \mathrm{l} & 0.88-2.01 \mathrm{~g} / \mathrm{l} \\ \text { C3 } & 17 \text { days after admission } & 1.01 \mathrm{~g} / \mathrm{l} & 0.88-2.01 \mathrm{~g} / \mathrm{l}\end{array}$

Table 2. Full blood count results for the patient prior to discharge.

$\begin{array}{lll}\text { Parameter } & \text { Value } & \text { Hospital normal range } \\ \text { White cell count } & 26.7 & 4-11 \times 10^{9} / / \\ \text { Neutrophils } & 7.7 & 2-7.5 \times 10^{9} / / \\ \text { Lymphocytes } & 15.8 & 1-4 \times 10^{9} / / \\ \text { Monocytes } & 3.5 & 0.2-1 \times 10^{9} / \mid \\ \text { Platelets } & 403 & 150-400 \times 10^{9} / \mid \\ \text { Haemoglobin } & 100 & 115-165 \times 10^{9} / \mid \\ \text { Mean corpuscular volume } & 81 \mathrm{fl} & 80-98 \mathrm{fl}\end{array}$

transient swelling of the deeper layers of the skin or mucous membranes of the upper respiratory or gastrointestinal tracts, and can be life threatening when the upper respiratory is involved. Though usually triggered by overwhelming histamine release, in rare cases bradykinin is responsible. Bradykinin-mediated angioedema should be considered in patients presenting without urticaria, ${ }^{1}$ to include hereditary angioedema (HAE) or acquired angioedema (AAE), although the most common cause of first presentations in middle-aged or elderly patients is ACE inhibitor use. Both HAE and AAE are characterised by excessive bradykinin release due to C1-INH deficiency and overactivation of the contact system, leading to impaired, uncontrolled classical complement pathway activation, angioedema and associated symptoms which may include abdominal pain. Data on prevalence of HAE and AAE in the UK is limited but there is probably a significant delay in diagnosis (up to 18 years for HAE or up to 5 years for AAE). ${ }^{2}$

Poor response to adrenaline and steroids in the initial stages of acute management should alert clinicians to the possibility of HAE or AAE, for which there is now growing evidence to show that steroids are ineffective. Acutely unwell patients will respond only to direct administration of plasma C1-INH concentrate, or icatibant (Table 3$).{ }^{3}$ Icatibant has been used for the management of acute attacks at a number of centres in the US, UK and Europe and is administered via prefilled syringe. ${ }^{4}$ The kallikrein inhibitor, ecallantide, has been used with some success in the US but is not currently licensed in Europe. ${ }^{5}$

Upper respiratory and gastrointestinal mucosal oedema caused by HAE or AAE warrants close monitoring by flexible nasendoscopy. Percutaneous tracheostomy in patients with compromised airways may be contraindicated due to severe soft tissue oedema. Consideration of surgical tracheostomy requires multidisciplinary-team input to determine timing of surgery in order to avoid exacerbating soft tissue oedema to the neck. Monitoring of mucosal oedema and treatment progress are facilitated by surgical tracheostomy and repeated flexible nasendoscopy.

In the long term, a detailed history is essential in determining underlying diagnosis. A positive family history points towards HAE. AAE should be considered in middle-aged or elderly patients without a family history of angioedema but with a past medical history of lymphoproliferative or autoimmune disease. The precise nature of the link between lymphoproliferative disorders and AAE is unclear, and either diagnosis may precede subsequent development of the other. ${ }^{6}$ Other clinical features should also be considered: besides the absence of urticaria,

Table 3. Current treatment options for acute and long-term management of bradykinin-mediated angioedema (in the UK).

\begin{tabular}{|c|c|c|c|}
\hline Medication & Indication & Dose/administration & Mechanism of action \\
\hline Plasma-derived C1 INH & Acute attacks & $20 \mathrm{IU} / \mathrm{kg}$ IV & $\begin{array}{l}\text { Direct inhibition of plasma kallikrein so that bradykinin } \\
\text { production is reduced }\end{array}$ \\
\hline Icatibant & Acute attacks & $\begin{array}{l}30 \text { mg subcutaneously, dosed } 6 \text { hours } \\
\text { apart }\end{array}$ & $\begin{array}{l}\text { Direct inhibition of plasma kallikrein so that bradykinin } \\
\text { production is reduced }\end{array}$ \\
\hline Tranexamic acid & Prophylaxis & $1-4.5 \mathrm{~g}$ daily in divided doses $\mathrm{PO}$ & $\begin{array}{l}\text { Antifibrinolytic agent to prevent plasminogen-plasmin } \\
\text { conversion, sparing } \mathrm{C} 1 \text {-INH in the process }\end{array}$ \\
\hline Danazol & Prophylaxis & 10-200 mg OD PO & Attenuated androgen - sparing effect on C1-INH use. \\
\hline
\end{tabular}

C1-INH $=$ C1 esterase inhibitor. 
Table 4. Complement test results typical of the different forms of angioedema. ${ }^{8}$

\begin{tabular}{llllll} 
Form of angiodema & C1 INH level & C1 INH function & C4 level & C3 level & C1Q level \\
HAE type 1 & Low & Low & Low & Normal & Normal \\
HAE type 2 & Normal-high & Low & Low & Normal & Normal \\
Acquired C1-INH deficiency & Low & Low & Low & Low-normal & Low \\
ACEi-triggered angioedema & Normal & Normal & Normal & Normal & Normal \\
Idiopathic angioedema & Normal & Normal & Normal & Normal & Normal \\
\hline ACEi = angiotensin-converting-enzyme inhibitor; C1-INH =C1 esterase inhibitor; HAE = hereditary angioedema. & & &
\end{tabular}

facial swelling is more commonly a feature of AAE, whereas limb and/or genital swelling may be more suggestive of HAE.? If HAE or AAE are suspected, referral to a specialist immunology team is advised for further investigation (including complement testing - Table 4). Long-term management of HAE and AAE focuses on treatment of underlying causes (eg lymphoproliferative disorders) and preventive therapy (see Table 3 ). The most appropriate preventive therapy is unclear, but there is growing evidence to support the use of antifibrinolytics (eg tranexamic acid) or attenuated androgens (eg danazol). ${ }^{7}$ Current guidance recommends provision of at least two $30 \mathrm{mg}$ icatibant pens to patients following appropriate training for immediate administration in the community if symptoms appear. A letter notifying clinical staff of the condition and best management should be carried by patients at all times.

\section{Learning points}

$>$ In rare cases, acute angioedema does not respond to conventional treatments with antihistamines and steroids. Acquired or inherited angioedema (C1-INH deficiency) should be considered as a differential diagnosis in these patients, especially in the absence of urticaria.

$>$ In acquired angioedema, there is usually no family history of angioedema, and quite often the patient is known to have an underlying lymphoproliferative disorder.

> Treatment with a bradykinin inhibitor or C1-INH substitute should be considered as first line therapy in these patients.

$>$ Long-term management revolves around patient education and treating the underlying causes where identified.

\section{References}

1 Jaiganesh T, Wiese M, Hollingsworth $\mathrm{J}$ et al. Acute angioedema: recognition and management in the emergency department. Eur $J$ Emerg Med 2013;20:10-7.

2 Jolles S, Williams P, Carne E et al. A UK national audit of hereditary and acquired angioedema. A UK national audit of hereditary and acquired angioedema. Clin Exp Immunol 2014;175:59-67.

3 Cicardi M, Zanichelli A. Acquired angioedema. Allergy Asthma Clin Immunol 2010;6:14-8.

4 Zanichelli A, Bova M, Coerezza A et al. Icatibant treatment for acquired C1-inhibitor deficiency: a real-world observational study. Allergy 2012;67:1074-7.

5 Drug Development Technology. Kalbitor - treatment for hereditary angioedema. 2014. Available online at www.drugdevelopmenttechnology.com/projects/dyax-corps-kalbitor/ [Accessed 22 September 2014].

6 Castelli R, Zanichelli A, Cicardi M, Cugno M. Acquired C1-inhibitor deficiency and lymphoproliferative disorders: a tight relationship. Crit Rev Oncol Hematol 2013;87:323-32.

7 Bouillet-Claveyrolas L, Ponard D, Drouet C, Massot C. Clinical and biological distinctions between type I and type II acquired angioedema. Am J Med 2003;115:420-1.

8 Zuraw BL, Bernstein JA, Lang DM et al. A focused parameter update: hereditary angioedema, acquired C1-esterase inhibitor deficiency, and angiotensin converting enzyme inhibitor-associated angioedema. J Allergy Clin Immunol 2013:131;1491-93.e25.

Address for correspondence: Dr S Ismail, Royal London Hospital, Whitechapel Road, London E1 1BB, UK. Email: sharif.ismail09@gmail.com 